\title{
3 Research Suare \\ Medical training alongside Covid-19: A qualitative analysis of the junior doctor experience
}

\section{Ciaran Grafton-Clarke ( $\nabla$ ciarang-c@hotmail.com )}

School of Medicine, University of Leicester, Leicester, UK https://orcid.org/0000-0002-8537-0806

\section{Hussein Uraiby}

University Hospitals of Leicester NHS Trust, Department of Clinical Education, UK

Shalin Abraham

University Hospitals of Leicester NHS Trust, Department of Clinical Education, UK

Joanne Kirtley

University Hospitals of Leicester NHS Trust, Department of Clinical Education, UK

Mark McCarthy

University Hospitals of Leicester NHS Trust, Department of Clinical Education, UK

\section{Research Article}

Keywords: medicine

Posted Date: July 26th, 2021

DOI: https://doi.org/10.21203/rs.3.rs-745131/v1

License: (c) (i) This work is licensed under a Creative Commons Attribution 4.0 International License.

Read Full License 


\section{Abstract \\ Background}

Covid-19 has had a seismic impact on medical training, in ways that are continually being realised. In response to the call to establish a 'new normal', we performed a detailed evaluation and thematic analysis of recent trainee experiences, which identifies the priorities for developing an innovative training framework that is adaptable during the peaks and troughs of a pandemic.

\section{Methods}

A 14-item survey was sent to junior doctors at a large teaching hospital. Data was analysed using a grounded theory framework and cycles of open and axial coding allowed for identification of emergent themes. Explanatory models were developed using inductive analysis to postulate thematic relationships.

\section{Results}

137 junior doctors responded to the survey. Respondents across all grades reported benefits to themselves, clinical teams and the profession. A notable benefit to junior trainees was the greater consultant support, fostering a more supportive work environment. Respondents also identified several challenges to working practice, including the availability of suitable PPE, burnout and the general pressures on physical and mental wellbeing.

\section{Discussion}

Trainees' perceived value was inferred typically from how 'busy' they were, possibly as postgraduate training is largely competence based. Educational bodies should attempt to highlight the equal value of non-technical skills, such as mentorship and problem-solving for a rounded curriculum. Trainees highlighted the inequity of opportunities due to sometimes inappropriate redeployment, suggesting that educational bodies should strive to develop personalised training programmes. Covid-19 has shown that a sense of personal physical and psychological safety to be of paramount importance to trainees.

\section{Introduction}

It is ubiquitous that the study of the pathophysiological mechanisms and widespread health consequences associated with the novel coronavirus (Covid-19) is a research priority.[1] The international research community has responded to this call to arms in a truly inspiring way, with the UK leading the charge in the key research strands of therapeutics,[2] immunology,[3] and epidemiological analysis.[4] Shrouded in uncertainty is the impact Covid-19 on medical education, for which the true impact on trainees, educators, and the wider medical education discourse, is yet to be fully determined. 
Modern medical education encompasses highly structured curricula whose architecture and requirements are specified by expert training bodies; integrated within training programmes to maintain an equilibrium between service provision and self-development; and guided by educators who have mastered their craft. [5] The Covid-19 pandemic was met by the UK government in a drastic manner, resulting in an unparalleled response aimed at reducing transmission and maintaining economic stability.[6] Many of these measures, such as the requirement to social distance, restriction of non-essential gatherings, and 'lockdown', have impacted seismically on the delivery of medical education. Furthermore, the unprecedented rise in patient numbers in acute clinical areas, shortages of personal protective equipment, [7] and concerns around exposure to asymptomatic carriers,[8] further descended the training of doctors to the lower tiers of organisational priority. Doctors in training were re-distributed to high acuity clinical areas, medical students were recruited as clinical assistants,[9] and pleas made to recently retired physicians to return to practice.[10] Doctors have been working greater number of hours, in more challenging clinical environments, whilst having to deal with the personal threat of Covid-19 on mental and physical health.[11] Accompanying rafts of workforce changes were mass cancellations and postponements of courses, conferences, formal teaching programmes, study leave, and post-graduate examinations, many of which are necessities for career progression. Additionally, trainees engaging with out of programme activities, such as research, education, and leadership, which are of great value to trainees and the future consultant NHS workforce, were driven back into clinical care, further compounding the complexity in restoration of normality.[12]

Several months into the disruption, correlating with easing of lockdown measures, focus is shifting towards creating a 'new normal'. What this 'new normal' looks like for medical education is currently evolving. Careful evaluation is required to guide the delivery of education through and beyond the current pandemic to ensure training programmes remain fit for purpose given the real-time changes in healthcare delivery.[13] It should be recognised that re-establishment of postgraduate medical education is complex, for which the principles of equity, safety, and effectiveness should be held as the grounding principles upon which we build. The starting point of this dynamic process is to discuss and reflect upon the experiences of junior doctors working at the bedside of those with Covid-19. The aim of this paper is to identify key themes and elucidate the meaning of such constructs, and to offer a series of priorities for those involved in postgraduate medical education to consider as we venture into the 'new normal'.

\section{Methods}

An anonymous online survey was developed and distributed to junior trainees working within a large University teaching hospital in the UK (Supplemental Material). The survey was sent to all junior doctors working within a formal training programme $(n=774)$, including the UK Foundation Programme, Core Medical Training (CMT), Core Surgical Training (CST), and speciality training

A 14-item survey was designed using a combination of dichotomous, multiple choice, Likert response scale, and free text questions. Surveys were sent to potential participants on the 2nd June 2020 via an introductory email, with a hyperlink directing the recipient to an online survey, executed by the web-based 
survey platform, KeySurvey. The survey closed on the 1st July 2020, with reminder emails sent every three days until closure.

The question schedule was developed through a multi-step process, and built upon a previously validated tool.[14] First, broad themes of interest were decided upon to ensure the constructs made theoretical sense within the scholarly discourse.[15] A scoping review of the literature informed this process, for which four key themes were chosen for the survey by consensus decision-making. These themes were: redeployment; training; rewards and successes; and challenges to working practice. Next, the items within the survey were developed in an iterative manner and written in accordance with current best practice in survey design, as to ensure representativeness of constructs, clarity, relevance, and distribution.[15] Finally, pilot testing was conducted to ensure adequacy of item variance, reliability, and convergent / discriminant validity.

A grounded theory analytic framework was applied to the data collected, but not applied in toto.[15 16] Iterative cycles of comparative analysis for emergent themes were performed using open and axial coding. ${ }^{14}$ This process was performed by two authors independently. This allowed for the consensual generation of interpretative theories within the boundaries of the pre-determined constructs. Theoretical sampling to saturation and iterative cycles of collection and analysis to shape data collection and the pursual of emergent themes, core components of grounded theory in toto, was not performed given the dynamic nature of the pandemic and imminent changeover of junior doctors to new clinical rotations. Subsequent inductive analysis towards the generation of explanatory models to articulate relationships between themes was performed.

The NHS Research Ethics Committee tool provided by the Medical Research Council determined that ethical approval was not required for this study.[17]

\section{Results}

137 junior doctors responded to the survey.

\section{Redeployment}

$22.6 \%$ of respondents had been redeployed to a different clinical area $(n=31)$. Trainees were redeployed to a number of different areas, which included intensive care $(25.9 \%)$, acute or emergency medicine $(19.4 \%)$, palliative care $(12.9 \%)$, cardiology or respiratory medicine $(12.9 \%)$, and other medical specialities (12.9\%).

Trainees reported a wide range of concerns they felt prior to redeployment. Most frequently, respondents were concerned about the safety of themselves and / or their family members $(n=16)$ and their personal capability to fulfil the role asked of them $(n=9)$. A small number were initially concerned about supervision $(n=2)$ or the impact of redeployment on their training $(n=3)$. 
Of those redeployed, $74.2 \%(n=23)$ felt their contribution following redeployment was worthwhile. Trainees reported worthwhileness relating to self and to the wider team. Respondents commonly reported on their perceived value within the team $(n=10)$, stating they felt 'unnecessary' as shifts were 'often very quiet'. A few trainees felt their value would have been greater if they remained in their parent clinical area. For instance, one trainee reported redeployment was to cover a pre-existing rota gap within a medical speciality and felt this was inappropriate given the burden their own parent speciality was under. A selection of trainees $(n=5)$ recognised that even though their own workload was not burdensome, the redeployment was worthwhile for the team since it relieved pressure on the existing staff and buffered the system to mitigate against sickness. A small number of trainees $(n=5)$ felt redeployment was worthwhile as it offered them opportunities to experience different aspects of medicine. One orthopaedic registrar described the opportunity to work within the proning team on ICU as 'humbling'; since it supported the strained ITU nurses and wider intensive care team in a time of crisis. While several respondents recognised their contribution to the 'pandemic effort' as worthwhile, no respondent explicitly reported on improvements to patient care.

\section{Training}

$55 \%$ of respondents $(n=76)$ had significant concerns about their future training. The nature of the concerns varied depending on the stage of training. For instance, foundation trainees had predominant concerns around competence, such as proceeding through training without undertaking a core placement, such as medicine or surgery. Additionally, foundation trainees were concerned about the loss of variation in clinical rotations, thus impacting their ability to make informed career choice decisions. Being a common theme across all trainee groups, the uncertainty around cancelled, rescheduled, and reformatted postgraduate examinations was a major concern. This uncertainty was underpinned by feelings of 'burnout' and a 'deterioration in motivation' when reflecting upon career progression.

Core trainees (or equivalent), were highly concerned about the significant loss of training opportunities, with the consequence of programme failure or necessitation for extension. They felt the marked reduction in training opportunities, namely procedural opportunities, courses summative to progression, and the opportunity to engage with research, quality improvement, and teaching, was affected, thus reducing their competitiveness when applying for jobs within a saturated marked. Speciality trainees commonly reported concerns around de-skilling, particularly in highly technical or procedural specialities, such as anaesthetics and surgery, resulting from the change in practice and training opportunities. A number of speciality trainees commented on systemic concerns throughout all areas of their training and development as a result of a perceived failure of (educators / educational bodies) to invest and recognise the importance and value of their training needs, meaning they 'will face uncertainty and disadvantage for the foreseeable future'. A matrix displaying the impact of Covid-19 on future training is presented in Fig. 1.

\section{Rewards and Successes}


Respondents across all grades of seniority and experience reported a spectrum of benefits to themselves, their clinical teams, and the profession as a whole, because of the Covid-19 pandemic. Predominately, participants commended the improvement in teamworking, morale, and 'flattening of the hierarchy', and the changing roles and responsibilities within the interdisciplinary team. Participants also felt the opportunity to contribute to 'the pandemic effort' as a major reward, with respondents stating: 'providing compassion', 'delivering high-quality care', and 'making a difference', were major motivators throughout the pandemic. The more junior trainees (foundation programme and core trainees) frequently reported the greater presence and involvement of their consultant colleagues to be a major driver in forging an environment of 'support', 'comradery' and 'fostering a culture of constructive feedback'. Whilst mentioned less frequently, responders report the increased attention directed towards staff wellbeing (e.g. provision of food, access to car parking facilities, rest facilities during shifts) has risen to the forefront of the agenda within the trust. A number of trainees made comments around the spotlight attention the pandemic has brought to the profession, reporting 'wide and universal public support for the National Health Service and its key workers' and the 'new found' respect the public has displayed towards medical personnel. Finally, several speciality trainees report on the unfettered 'innovation and new modes of practice' as an exciting opportunity for healthcare to be more 'versatile', 'dynamic and adaptable', and 'technology-focused'.

\section{Challenges to Working Practices}

Junior doctors working through the Covid-19 pandemic experienced challenges to their practice across several domains. The availability and safety of PPE was a major issue. Firstly, numerous trainees reported inadequate provisions and appropriate fit-testing sessions; 'claustrophobia' related to its use; and its 'impact on the ability to communicate clearly and freely with patients, colleagues, and relatives'. Several respondents described the isolating effect of having to remove themselves from their family (in the case of shielding / vulnerable members of a household), and the wider burden the pandemic placed upon their physical and mental wellbeing. For example, several trainees cited the 'emotional impact of patients dying' as a significant personal challenge. Others described feeling 'burnout resulting from observing the horrific aspects associated with the pandemic'. One respondent felt as if they were 'living in constant fear of taking Covid-19 home to the family'.

\section{Discussion}

\section{Analytic approach}

This study draws on the experiences and reflections of a cohort of junior doctors working on the 'frontline' of the Covid-19 pandemic. Amongst the tales of heroism, professional anguish and calls for restitution are several important inter-digitating themes underpinning the expression of concern and personal need from doctors within a training programme. It is through consideration of such themes within the context of social science and educational theory can meaning be inferred.[15] It is this critical appraisal amongst the richness of information within qualitative methodology,[18] that can inform the wider discourse as to 
facilitate learning and progression towards establishment of a 'new normal'. A 'new normal' whereby our trainee doctors are cared for and valued; provided with education and training permissive for development; and are free to raise concerns about any aspect of their working lives.[19]

\section{Perception of value: what matters?}

Junior doctors are not averse to contributing in significant ways to the operations and leadership of the teams in which they work. They are integral to the delivery of healthcare to the population, for which they are duly accomplished at balancing service provision with their education and development. They are innately resourceful, imaginative, organised, and focused, which adds significant value to their clinical teams. From an external perspective, the value of junior doctors is immense across the breadth of clinical practice; but how do they themselves gauge their value? It is apparent from this study that self-perceived value is typically related to how 'busy / involved' a junior feels within their role, for which trainees frequently reported their purpose as 'pointless' if there was a low patient burden or caseload within their clinical area. In contrast, trainees working within busy clinical environments, frequently described their contributions using language associated with battles and tremendous efforts in the face of adversity, such as 'the pandemic effort' and 'against the fatal disease'. The more subtle contributions made by a trainee upon their clinical teams are clearly held in lower regard compared to this overt expression of contribution. Elements such as mentorship, problem-solving, continuity of care, and commitment to clinical excellence, whilst more abstract in measurability, are major contributions made by junior doctors both within and outside the pandemic; but were not considered by trainees in their assessment of value. Why is this the case?

We theorise that since post-graduate training is largely competence-based, for which trainees are encouraged to demonstrate technical capability and knowledge through a range of assessments, such as Case-Based Discussions (CBD) and Direct Observations of Procedural Skills (DOPS), all of which require direct patient contact, less emphasis is placed on the broader contributions made by trainees. Considering this, the frustration demonstrated by redeployed trainees in 'less busy' clinical environments become understandable, particularly if they feel their colleagues are receiving greater exposure to opportunities conductive towards portfolio completion. This reinforces the need for trainers and educational / training bodies to reveal and accentuate the importance of non-technical skills, as to facilitate widespread acceptance that training a doctor extends far beyond achievement of competencies. [20]

\section{Training is an absolute priority to junior trainees}

Postgraduate training for a doctor is a highly personal process and requires enormous investment in terms of commitment, finance, and time.[21] Ranging from repeated upheaval and re-location, to having to progress through series of challenging examinations, through to demonstrating competence by means of assessed portfolios; the impact of training can transmit to all spheres of a doctor's life.[22] It is therefore unsurprising that even amid a global pandemic that training needs are highlighted as a major area of concern. While junior doctors are better placed than most to comment on the devastating impact 
Covid-19 has had upon the safety of our population, why has the issue of reduced training opportunities / potential for delayed progression been raised as such an emotive issue within our survey? On paper, the maximal impact of Covid-19 upon training is around extending the length of training to ensure all the necessary competencies have been achieved, which itself may be advantageous to a trainee in ensuring they are capable of independent practice once they obtain their Certificate of Completion of Training (CCT). It is undeniable that working at the bedside of those with Covid-19 has offered trainee doctors an education like no other. Resilience, humility, and communicating with empathy, often forgotten amongst the drill and strain of modern clinical practice, have become the pillars of our clinical teams throughout the pandemic. Our profession will benefit immeasurably from the increased awareness of dignity and end of life care,[23] importance of communicating with openness in physically and emotionally challenging situations, and the need to prioritise our personal wellbeing.[24] Despite this recognition of benefit, why have issues around training, opportunities, and progression been raised with such rancour? We theorise an inter-play of factors, including inequity of opportunity, individuality of training, and lack of investment by trainers and educationalists.

The strategy of workforce redeployment, common to most healthcare trusts nationally, was largely based on perception and not based on predictive modelling, intimate monitoring of hospital activity, or widespread consultation. While this was understandable given the emergent nature of the pandemic, it unfortunately contributed to inequity in opportunity amongst trainees. The distribution of hospital activity shifted markedly from outpatient and elective services towards the delivery of acute care.[25] This necessitated redeployment of some trainees away from their parent clinical areas,[6] leaving the remnant trainees to deliver skeleton services, which in some cases offered them greater than usual exposure to their speciality. This unintentional inequity may be in part responsible for the expression of concern raised by a sizeable proportion of redeployed trainees. Linked closely to this, is the concept of postgraduate training being a highly individualised entity. At any given point in time, individual trainees require investment in different areas of their development, whether this be number of cases, exposure to a certain case-mix, demonstration of specific procedural or surgical competencies. Therefore, the failure to consider trainees as individuals within the redeployment strategy is likely to have further contributed to the disadvantage faced by certain trainees. It is this lack of consideration and 'visibility of trainer involvement' to mitigate against disadvantage, which has drawn trainees to express grave concerns about the future of medical training within the 'new normal'. Whilst adjustment of portfolio and progression requirements as to not disadvantage any individual trainee has been widely performed,[26] it is essential each trainee is offered a needs-based assessment of their development. This is important for two reasons. Firstly, to ensure all trainees receive personalised programmes of training to ensure the unintended inequity experienced by some is not transmitted through the post-Covid era. Secondly, the confidence and faith placed in trainers, programme leads, and training bodies, by trainees is of paramount importance, and efforts should be made to foster a dialogue of openness, understanding, and flexibility.

\section{Covid-19 as a personal threat}


The Covid-19 pandemic felt very personal to healthcare professionals. Very few have been in the position where they have had to deliver intensive medical therapy to individuals previously known as colleagues. Within a short period of time, seemingly robust and well individuals required the most invasive of lifepreserving therapies. Hundreds of healthcare workers have died at the hands of Covid-19 in the UK.[27] It is this ominous reality, in conjunction with the widely published issues around the provision and safety of PPE, that instilled a sense of anxiety and anger within the profession.[28] Subsequent evidence highlighting the increased risk faced by Black, Asian and minority ethnic groups (BAME), further escalated concern.[29] Sense of personal safety is clearly an absolute priority for junior doctors, and links intimately with interpretation of value and disposability. Comparatively, concerns about personal safety over-shadowed the infrequent concern recorded for patients. The unwavering desire of trainees to be cared for and valued should be recognised as an institutional priority. Analogous to Maslow's hierarchy of need,[30] a similar model describing the motivational needs of junior trainees has been produced, reiterating the importance of supporting trainees holistically (Fig. 2).

\section{What this study adds}

This study contributes to the scholarly discourse as it expands upon a range of frequently cited barriers and challenges faced by junior doctors. The strength in this review lies in its analytical approach, for which the originality, resonance, and usefulness of its results are transferrable across the breadth of postgraduate training. This study offers new insights into the complexities of delivering training through and beyond Covid-19 and captures the essence and fullness of the junior trainee experience. Furthermore, the systematicity within the collaborative interpretative process ensured a balanced render of the data. Whilst this study involved participants from a single centre, it is the generation of conceptional understandings that are transferrable and useful for educators across the nation.

\section{Conclusion}

The Covid-19 pandemic represents the greatest disruption to the delivery of medical education in recent history. While our junior doctors have been instrumental in the delivery of high-quality care, it is now time for us to appraise the impact of the pandemic on training. Medical education and the training of doctors will no doubt change within the era of the 'new normal'. This study offers educators insight into the issues facing trainee doctors and delivers a framework that should be built upon in order to individualise education and training going forward. It is through making sense of the experiences and reflections of trainees can we start preparing for what is to come next.

\section{Lessons For Practice}

1. Educational bodies should attempt to highlight the equal value of non-technical skills, such as mentorship and problem-solving for a rounded curriculum.

2. Trainees highlighted the inequity of opportunities due to sometimes inappropriate redeployment, suggesting that educational bodies should strive to develop personalised training programmes. 
3. The confidence and faith placed in trainers, programme leads, and training bodies, by trainees is of paramount importance, and efforts should be made to foster a dialogue of openness, understanding, and flexibility.

\section{Declarations}

\section{Conflicts of Interest}

No conflicts of interest to declare.

\section{Funding}

No funding received for this research work.

\section{Acknowledgements}

With thanks to Mr Luke Ruffle for his support in conducting the survey.

\section{Ethics}

No formal ethical approval required.

\section{References}

1. Wu F, Zhao S, Yu B, et al. A new coronavirus associated with human respiratory disease in China. Nature 2020;579(7798):265-69 doi: 10.1038/s41586-020-2008-3[published Online First: Epub Date].

2. Horby P, Lim WS, Emberson J, et al. Effect of Dexamethasone in Hospitalized Patients with COVID-19: Preliminary Report. medRxiv 2020:2020.06.22.20137273 doi: 10.1101/2020.06.22.20137273[published Online First: Epub Date]|.

3. Folegatti PM, Ewer KJ, Aley PK, et al. Safety and immunogenicity of the ChAdOx1 nCoV-19 vaccine against SARS-CoV-2: a preliminary report of a phase $1 / 2$, single-blind, randomised controlled trial. The Lancet 2020;396(10249):467-78 doi: 10.1016/S0140-6736(20)31604-4[published Online First: Epub Date]|.

4. Anderson RM, Hollingsworth TD, Baggaley RF, et al. COVID-19 spread in the UK: the end of the beginning? The Lancet 2020 doi: 10.1016/S0140-6736(20)31689-5[published Online First: Epub Date]|.

5. Council GM. Promoting excellence: standards for medical education and training. 2015

6. Office NA. Overview of the UK government's response to the COVID-19 pandemic. 2020 
7. Rimmer A. Covid-19: Third of surgeons do not have adequate PPE, royal college warns. BMJ 2020;369:m1492 doi: 10.1136/bmj.m1492[published Online First: Epub Date]|.

8. Yu X, Yang R. COVID-19 transmission through asymptomatic carriers is a challenge to containment. Influenza Other Respir Viruses 2020;14(4):474-75 doi: 10.1111/irv.12743[published Online First: Epub Date]|.

9. Rose S. Medical Student Education in the Time of COVID-19. JAMA 2020;323(21):2131-32 doi: 10.1001/jama.2020.5227[published Online First: Epub Date]|.

10. Service NH. Redeploying your secondary care medical workforce safely. 2020

11. The L. COVID-19: protecting health-care workers. The Lancet 2020;395(10228):922 doi: 10.1016/S0140-6736(20)30644-9[published Online First: Epub Date]|.

12. Macdougall C DP, Katz D, Strain W. The impact of COVID-19 on Medical education and Medical Students. How and when can they return to placements? MedEdPublish 2020;9(1):159

13. Kanneganti A, Sia C-H, Ashokka B, et al. Continuing medical education during a pandemic: an academic institution's experience. Postgraduate Medical Journal 2020;96(1137):384 doi: 10.1136/postgradmedj-2020-137840[published Online First: Epub Date]|.

14. Schönrock-Adema J, Visscher M, Raat ANJ, et al. Development and Validation of the Scan of Postgraduate Educational Environment Domains (SPEED): A Brief Instrument to Assess the Educational Environment in Postgraduate Medical Education. PLOS ONE 2015;10(9):e0137872 doi:

10.1371/journal.pone.0137872[published Online First: Epub Date]|.

15. Artino AR, Jr., La Rochelle JS, Dezee KJ, et al. Developing questionnaires for educational research: AMEE Guide No. 87. Med Teach 2014;36(6):463-74 doi: 10.3109/0142159X.2014.889814[published Online First: Epub Date]|.

16. Allen M. The SAGE Encyclopedia of Communication Research Methods. 2017 doi: 10.4135/9781483381411[published Online First: Epub Date]|.

17. Authority NHR. Do I need NHS REC review? 2020

18. Kennedy TJ, Lingard LA. Making sense of grounded theory in medical education. Med Educ 2006;40(2):101-8 doi: 10.1111/j.1365-2929.2005.02378.x[published Online First: Epub Date]|.

19. Kachra R, Brown A. The new normal: Medical education during and beyond the COVID-19 pandemic. Canadian Medical Education Journal 2020(0) doi: 10.36834/cmej.70317[published Online First: Epub Date]|. 
20. Patel M. Changes to postgraduate medical education in the 21 st century. Clinical Medicine 2016;16(4):311 doi: 10.7861/clinmedicine.16-4-311[published Online First: Epub Date]|.

21. Rich A, Viney R, Needleman S, et al. 'You can't be a person and a doctor': the work-life balance of doctors in training-a qualitative study. BMJ Open 2016;6(12):e013897-e97 doi: 10.1136/bmjopen-2016013897[published Online First: Epub Date]|.

22. Woolf K, Rich A, Viney R, et al. Perceived causes of differential attainment in UK postgraduate medical training: a national qualitative study. BMJ Open 2016;6(11):e013429-e29 doi: 10.1136/bmjopen-2016013429[published Online First: Epub Date]|.

23. Radbruch L, Knaul FM, de Lima L, et al. The key role of palliative care in response to the COVID-19 tsunami of suffering. The Lancet 2020;395(10235):1467-69 doi: 10.1016/S0140-6736(20)309648[published Online First: Epub Date].

24. Unadkat S, Farquhar M. Doctors' wellbeing: self-care during the covid-19 pandemic. BMJ 2020;368:m1150 doi: 10.1136/bmj.m1150[published Online First: Epub Date]|.

25. Hourston GJM. The impact of despecialisation and redeployment on surgical training in the midst of the COVID-19 pandemic. Int J Surg 2020;78:1-2 doi: 10.1016/j.ijsu.2020.03.082[published Online First: Epub Date]|.

26. Physicians RCo. Trainees and Covid-19. 2020

27. Cook T KE, Lennane S. Deaths of NHS staff from covid-19 analysed. 2020

28. Horton R. Offline: COVID-19 and the NHS - "a national scandal". The Lancet 2020;395(10229):1022 doi: 10.1016/S0140-6736(20)30727-3[published Online First: Epub Date]|.

29. Rimmer A. Covid-19: Two thirds of healthcare workers who have died were from ethnic minorities. BMJ 2020;369:m1621 doi: 10.1136/bmj.m1621[published Online First: Epub Date]|.

30. Maslow A. A Theory of Human Motivation. Psychological Review 1943;50:370 - 96

\section{Figures}




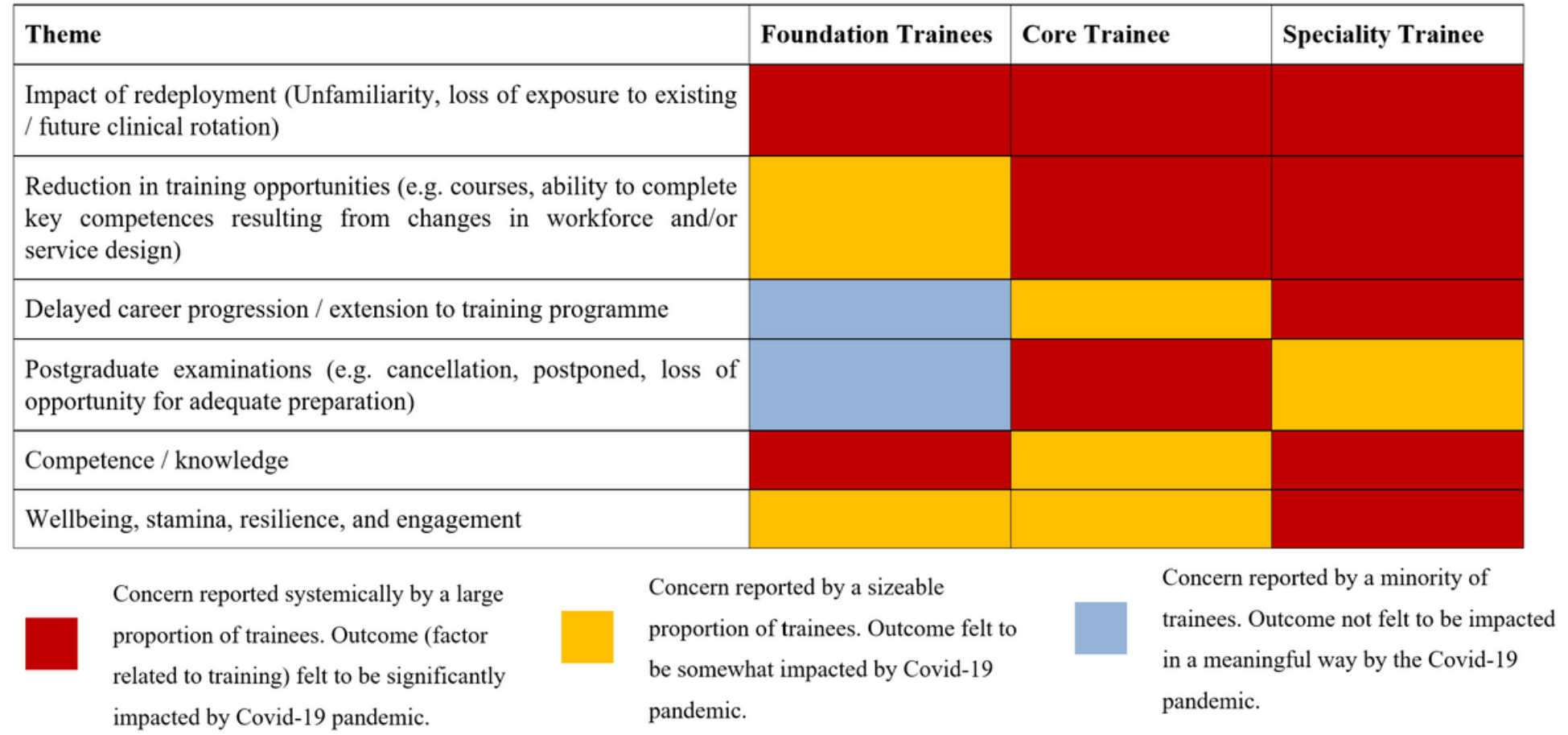

\section{Figure 1}

Matrix of the trainee-reported impact of Covid-19 on future training 


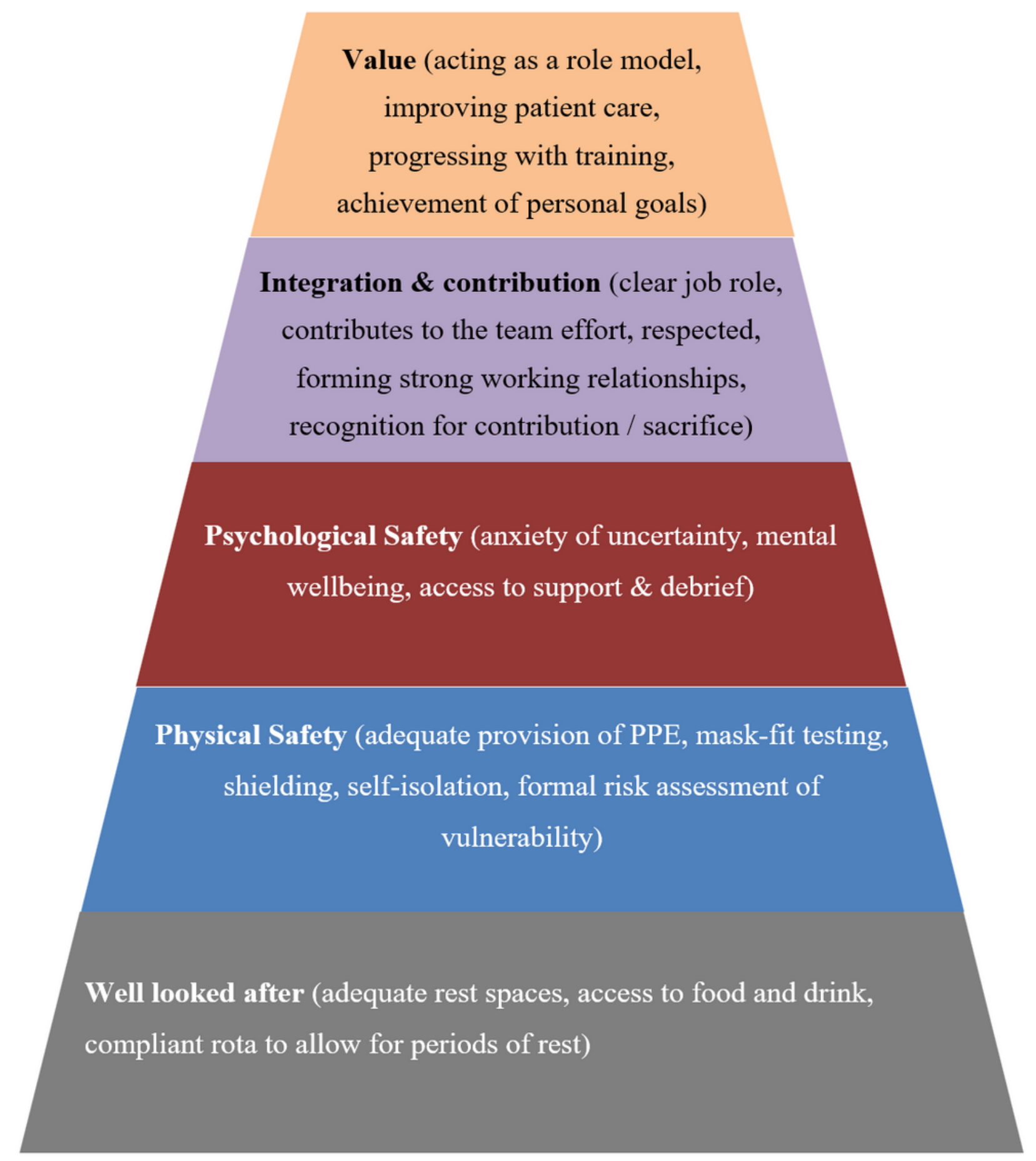

Figure 2

Motivational needs of trainee doctors

\section{Supplementary Files}

This is a list of supplementary files associated with this preprint. Click to download. 
- SupplementalMaterial.docx

Page 15/15 